\title{
The circular RNA expression profile in ovarian serous cystadenocarcinoma reveals a complex circRNA-miRNA regulatory network
}

Minhui Zhuang ${ }^{1 \dagger}$, Jian Zhao ${ }^{1 \dagger}$, Jing $\mathrm{Wu}^{1,2}$, Shilong $\mathrm{Fu}^{3^{*}}$, Ping $\mathrm{Han}^{3^{*}}$ and Xiaofeng Song ${ }^{1 *}$ (])

From The International Conference on Intelligent Biology and Medicine (ICIBM) 2020 Virtual. 9-10 August 2020

\begin{abstract}
Background: Ovarian serous cystadenocarcinoma is one of the most serious gynecological malignancies. Circular RNA (circRNA) is a type of noncoding RNA with a covalently closed continuous loop structure. Abnormal circRNA expression might be associated with tumorigenesis because of its complex biological mechanisms by, for example, functioning as a microRNA (miRNA) sponge. However, the circRNA expression profile in ovarian serous cystadenocarcinoma and their associations with other RNAs have not yet been characterized. The main purpose of this study was to reveal the circRNA expression profile in ovarian serous cystadenocarcinoma.
\end{abstract}

Methods: We collected six specimens from three patients with ovarian serous cystadenocarcinoma and adjacent normal tissues. After RNA sequencing, we analyzed the expression of circRNAs with relevant mRNAs and miRNAs to characterize potential function.

Results: 15,092 unique circRNAs were identified in six specimens. Approximately $46 \%$ of these circRNAs were not recorded in public databases. We then reported 353 differentially expressed circRNAs with oncogenes and tumor-suppressor genes. Furthermore, a conjoint analysis with relevant mRNAs revealed consistent changes between circRNAs and their homologous mRNAs. Overall, construction of a circRNA-miRNA network suggested that 4 special circRNAs could be used as potential biomarkers.

Conclusions: Our study revealed the circRNA expression profile in the tissues of patients with ovarian serous cystadenocarcinoma. The differential expression of circRNAs was thought to be associated with ovarian serous cystadenocarcinoma in the enrichment analysis, and co-expression analysis with relevant mRNAs and miRNAs illustrated the latent regulatory network. We also constructed a complex circRNA-miRNA interaction network and then demonstrated the potential function of certain circRNAs to aid future diagnosis and treatment.

*Correspondence: xfsong@nuaa.edu.cn; hanping200701@163.com;

docfusl@163.com

${ }^{\dagger}$ Minhui Zhuang and Jian Zhao are co-first authors

${ }^{1}$ Department of Biomedical Engineering, Nanjing University

of Aeronautics and Astronautics, Nanjing 211106, China

${ }^{3}$ Department of Gynecology and Obstetrics, The First Affiliated Hospital

With Nanjing Medical University, Nanjing 210029, China

Full list of author information is available at the end of the article

(c) The Author(s) 2021. Open Access This article is licensed under a Creative Commons Attribution 4.0 International License, which permits use, sharing, adaptation, distribution and reproduction in any medium or format, as long as you give appropriate credit to the original author(s) and the source, provide a link to the Creative Commons licence, and indicate if changes were made. The images or other third party material in this article are included in the article's Creative Commons licence, unless indicated otherwise in a credit line to the material. If material is not included in the article's Creative Commons licence and your intended use is not permitted by statutory regulation or exceeds the permitted use, you will need to obtain permission directly from the copyright holder. To view a copy of this licence, visit http://creativecommons.org/licenses/by/4.0/. The Creative Commons Public Domain Dedication waiver (http://creativecommons.org/publicdomain/zero/1.0/) applies to the data made available in this article, unless otherwise stated in a credit line to the data. 
Keywords: circRNA, Expression profile, Interaction network, Ovarian serous cystadenocarcinoma

\section{Background}

Ovarian cancer is a common type of tumor formed in the female reproductive system that poses a serious threat to the health of women [1]. Ovarian serous cystadenocarcinoma is an epithelial subtype of ovarian cancer that is difficult to cure. Because of the lack of effective screening technology and inconspicuous early-stage symptoms, diagnosis is often not made until advanced stages [2]. There is thus a need to develop more accurate methods for early detection. Researchers have already noticed that some features at the molecular level might provide signs of underlying illness. For example, family history was thought to indicate risk for ovarian cancer, which might be caused by genomic mutations [3]. Moreover, dysregulation of special genes associated with adverse prognosis in ovarian cancer, such as p53 and VEGF, suggested that they have the potential to be used as biomarkers in diagnosis and treatment $[4,5]$. However, the molecular mechanisms underlying ovarian serous cystadenocarcinoma remain unclear.

Circular RNA (circRNA) is a type of noncoding RNA with a special molecular structure. Although they were first discovered from RNA viruses over forty years ago, circRNAs were not confirmed to exist in human cells until 1991 [6-8]. circRNAs are different from their homologous linear transcripts in splicing forms, as they are typically formed by the back-splicing of exons from precursor mRNA (pre-mRNA). This procedure permits circRNAs to exist as RNA circles without polyadenylated (polyA) tails. In some cases, circRNAs can be relatively long-lived and weakly expressed [9]. In other cases, circRNAs can accumulate when they were abundant at a steady-state level post-transcriptionally [10]. Researchers have also recently found that the abnormal expression of circRNAs might be involved in human diseases. The abundance of circRNAs in colorectal cancer cell lines has been reported to be globally reduced, and a negative correlation between global circRNA abundance and proliferation has been validated for several diseases, including ovarian cancer [11]. In addition, some studies have suggested that circRNAs provide important indicators with prognostic implications. Hsa_circ_0001649 has been reported to be downregulated and associated with tumor size and differentiation grade in cholangiocarcinoma, indicating its potential as a cancer-related therapeutic target [12]. Another circRNA cSMARCA5 was suggested to provide a potential therapeutic target of hepatocellular carcinoma because it could mediate TIMO3 expression and inhibit the migration and proliferation of hepatocellular carcinoma cells [13]. In ovarian cancer, Ning et al. found that circEXOC6B and circN4BP2L2 can act as potential prognostic biomarkers [14]. Although several studies of circRNAs have been conducted, there is still a need for additional research to explore the mechanisms underlying human diseases.

The function of circRNAs in tumorigenesis might be associated with their complex biological mechanisms. At the expression level, circRNAs have been found to compete with linear splicing and act as regulators [15]. Another remarkable function of circRNAs is their ability to participate in regulation as microRNA (miRNA) sponges. For example, ciRS-7/CDR1as (CDR1 antisense) is often noted to function as a sponge of miR-7. This single-exon circularized RNA was determined to contain over 60 selectively conserved miRNA binding sites [16, 17] that allow it to function as a negative regulator for miR-7 through competitive combination and then modulate the expression of oncogenes targeted by mir-7 [18]. Moreover, testis-specific circRNA Sry (sex-determining region $\mathrm{Y}$ ) has been observed to function as a sponge of miR-138, which could inhibit cell migration and invasion in ovarian and breast cancer cells by targeting SOX4 and HIF- $1 \alpha[15,19]$. Therefore, the regulatory network related to circRNAs may help clarify tumorigenesis in ovarian serous cystadenocarcinoma.

Here, a transcriptome analysis was conducted to characterize the function of circRNAs associated with ovarian serous cystadenocarcinoma. We detected the differential expression of circRNAs in several tissues. Furthermore, a co-expression analysis with relevant mRNAs and miRNAs revealed several special circRNAs that could serve as potential biomarkers.

\section{Methods \\ Specimen collection and RNA sequencing}

We collected six specimens from three patients with ovarian serous cystadenocarcinoma. Three tumor specimens came from tumor tissues, and the other three normal specimens came from para-tumor tissues. All patients were informed of the purpose of the study and signed informed consents; the collection of specimens was approved by the ethics committee of Jiangsu Province Hospital. Total RNA from each specimen was extracted using the RNAsimple total RNA kit (Tiangen, China) per the manufacturer's instructions. cDNA fragments of 250-300 bp were isolated and then subjected to approximately 20 cycles of PCR amplification. For end repair and joint connection, we used the Illumina TruSeq 
total RNA library preparation kit. After further purification and quality detection of the amplified products, the total RNA library preparation was completed. Transcriptome sequencing was performed using HiSeq4000 (Illumina).

\section{Identification of human circRNAs and mRNAs}

To identify circRNAs and mRNAs, several pipelines were used. We used BWA to build the index of the reference genome and BWA-MEM, and the alignment algorithm was implemented as a component of BWA to map the paired-end reads of each specimen to the human reference genome [20]. The FASTA-formatted sequence file of the human reference genome was downloaded from the UCSC Genome Browser (GRCh37/hg19) [21]. We then used CIRI2 to identify circRNAs in tissues [22, 23]. Based on multiple seed matching, CIRI2 can use an adapted maximum likelihood estimation to identify backspliced junction reads. Based on the mRNAs in tissues, the expression level was evaluated with Tophat 2 and Cufflinks [24, 25]. Cufflinks can assemble transcripts and evaluate their abundances from aligned RNA-Seq reads after mapping by Tophat 2 .

\section{Dataset for bioinformatic analysis}

Data on known circRNAs were downloaded from circBase (H.sapiens updated in July 2017) [26]. For the co-expression analysis with miRNAs, experimentally validated tumorigenesis miRNAs were collected from miR2Disease (http://www.mir2disease.org/) together with their references [27]. Meanwhile, human miRNAtarget interactions (MTIs) were directly downloaded from miRTarBase Release 7.0 (http://mirtarbase.mbc. nctu.edu.tw/) [28]. miRTarBase provides several validated MTIs from collected research articles related to functional studies of miRNAs. Furthermore, genes validated from reporter assays are generally thought to provide strong evidence for interaction with miRNAs. In the end, data on human oncogenes and tumor-suppressor genes were collected from two public databases: ONGene and TSGene $2.0[29,30]$

\section{Functional enrichment analysis}

To conduct the functional enrichment analysis, we used Metascape, which is a set of reliable and intuitive tools that can obtain gene annotations and can be used to conduct gene list enrichment analyses (last update date: 2018-11-20) [31]. Metascape integrates a few related databases and provides a more comprehensive and updated enrichment analysis for gene lists. Standard accumulative hypergeometric statistical tests were used, and results were ordered by their $\mathrm{p}$-values.

\section{Construction of a circRNA-miRNA network}

In the co-expression analysis with related miRNAs, the miRanda pipeline was used to predict the interactions between miRNAs and circRNAs [32]. MiRanda provides a miRNA target scanner using dynamic-programming alignments and thermodynamics for assessments. Furthermore, miRNAs experimentally validated in connection with ovarian cancer were used to construct the circRNA-miRNA network.

\section{Results}

\section{Landscape of circRNAs in ovarian serous cystadenocarcinoma}

Overall, we found 15,092 unique circRNAs with at least 2 junction reads spanning a head-to-tail splice junction. A total of 6500,4370 , and 2774 circRNAs were identified in the three tumor specimens, and 6201, 4932, and 5606 circRNAs were found in the three normal specimens (Fig. 1A). And there was no correlation between sequence depths and numbers of detection (Additional file 1: Fig. S1). A total of 446 unique circRNAs appeared in the six specimens. Overall, 9543 unique circRNAs in tumor tissues and 10,358 in normal tissues shared the intersection composed of 4809 individuals (Fig. 1B). Furthermore, the proportion of novel individuals indicated that the circRNAs identified in these specimens were worth studying (Fig. 1C). We searched the identified circRNAs using circBase and found that nearly half of these circRNAs were not recorded in this database. We then calculated the proportion of different formation types of circRNAs. As expected, the majority of circRNAs (approximately 83\%) were annotated with the type of exon (Fig. 1D).

Given the above results, we further explored the abundance of circRNAs in different tissues. The distribution of circRNAs on human chromosomes is shown as bar charts (Fig. 1E). All circRNAs were distributed on each chromosome separately (except allosome Y); the differences were plotted in green bars for clarity. Despite the fact that there were more circRNAs in tumor tissues on some chromosomes, we still noticed that the numbers of circRNAs per chromosome in normal tissues were generally larger. Meanwhile, circRNAs were found to be especially enriched on the first three chromosomes.

(See figure on next page.)

Fig. 1 A Intersections of identified circRNAs in six specimens. B Overlap of identified circRNAs in tumor and normal groups. C Distribution of circRNAs with their annotations. D Distribution of circRNAs according to formation type. E Distribution of circRNAs on 23 human chromosomes (without chrY in ovarian tissues). Difference = number of circRNAs in tumor tissues—number of circRNAs in normal tissues. $\mathbf{F}$ Comparison of isoform number per gene between tumor and normal groups. G Distribution of circRNAs ordered by the number of back-spliced reads 


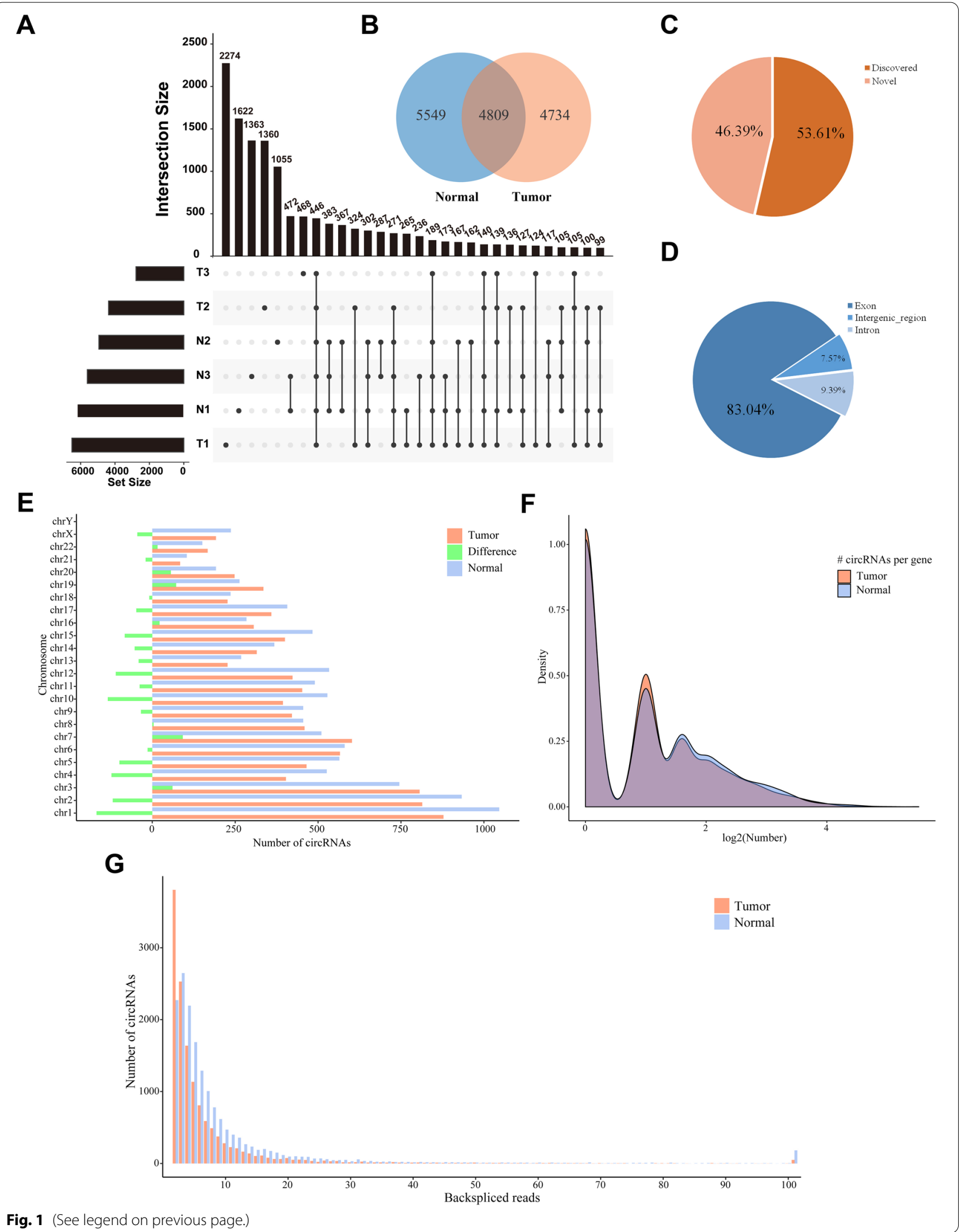


A possible reason for this observation was that the first three autosomes were relatively long. We then calculated the numbers of circRNA isoforms per gene (Fig. 1F). Overall, the normal tissues tended to have more homologous circular transcripts, although the gene PATK2 in tumor tissues had the most (46). The range was from 0 to 5, and most circRNAs in tumor tissues tended to have one or no homologous isoform. We also determined the distribution based on the calculated numbers of backspliced reads (with number $=1$ ignored). The number of circRNAs in the tumor group was unexpectedly larger than the other when the number of back-spliced reads was 2 (Fig. 1G). Despite the fact that the total number of circRNAs in normal tissues was larger, tumor tissues tended to amplify the low expression level of some circRNAs. As mentioned above, the expression patterns of circRNAs were clearly different between tumors and normal tissues.

\section{Differential expression of circRNAs in human tissues}

To further assess the function of circRNAs in human tissues, we performed differential expression analysis. Quantification of circRNAs was conducted using the number of back-spliced reads and then was normalized by RPM (reads per million mapped reads). In terms of expression, more circRNAs were downregulated in tumor tissues. Categorization was performed using a volcano plot (Fig. 2A). Moreover, data obtained after the stability assessment were used to examine differences between tissues. Some of the circRNAs were only significantly expressed in all three specimens of the tumor group or normal group. These group-specific species could also explain the function of circRNA. To highlight the differences, the expression level of the selected circRNAs mentioned above are shown in the heatmap (Fig. 2B).

Based on the screening, enrichment analysis was conducted with Metascape. Parent genes of circRNAs were used to conduct separate searches for relevant functions. Because of the disparity between the numbers in the two groups, the results of the enrichment analysis also revealed a major difference in the number of terms. Representative enriched terms of the top 9 clusters in the upregulated group tended to be GO biological processes focused on growth and regulation (Fig. 3A). GO:0031330: negative regulation of cellular catabolic process and GO:0045927: positive regulation of growth indicated that these circRNAs were possibly related to the abnormal growth and degradation of tumor cells. In addition, R-HSA-1257604: PIP3 activates AKT signaling had slightly more counts in the enriched terms. This pathway might reflect a more direct relationship to ovarian serous cystadenocarcinoma. Over the past few years, several studies have explored the connection between $\mathrm{AKT} / \mathrm{pAKT}$ and the invasion and metastasis of ovarian cancer [33]. In a study of FSH-stimulated VEGF expression in ovarian serous cystadenocarcinoma, the activation of the PI3K/AKT pathway mediated the upregulated expression of survivin and then promoted VEGF expression [34]. Moreover, positive AKT and phosphorylated AKT (pAKT) protein

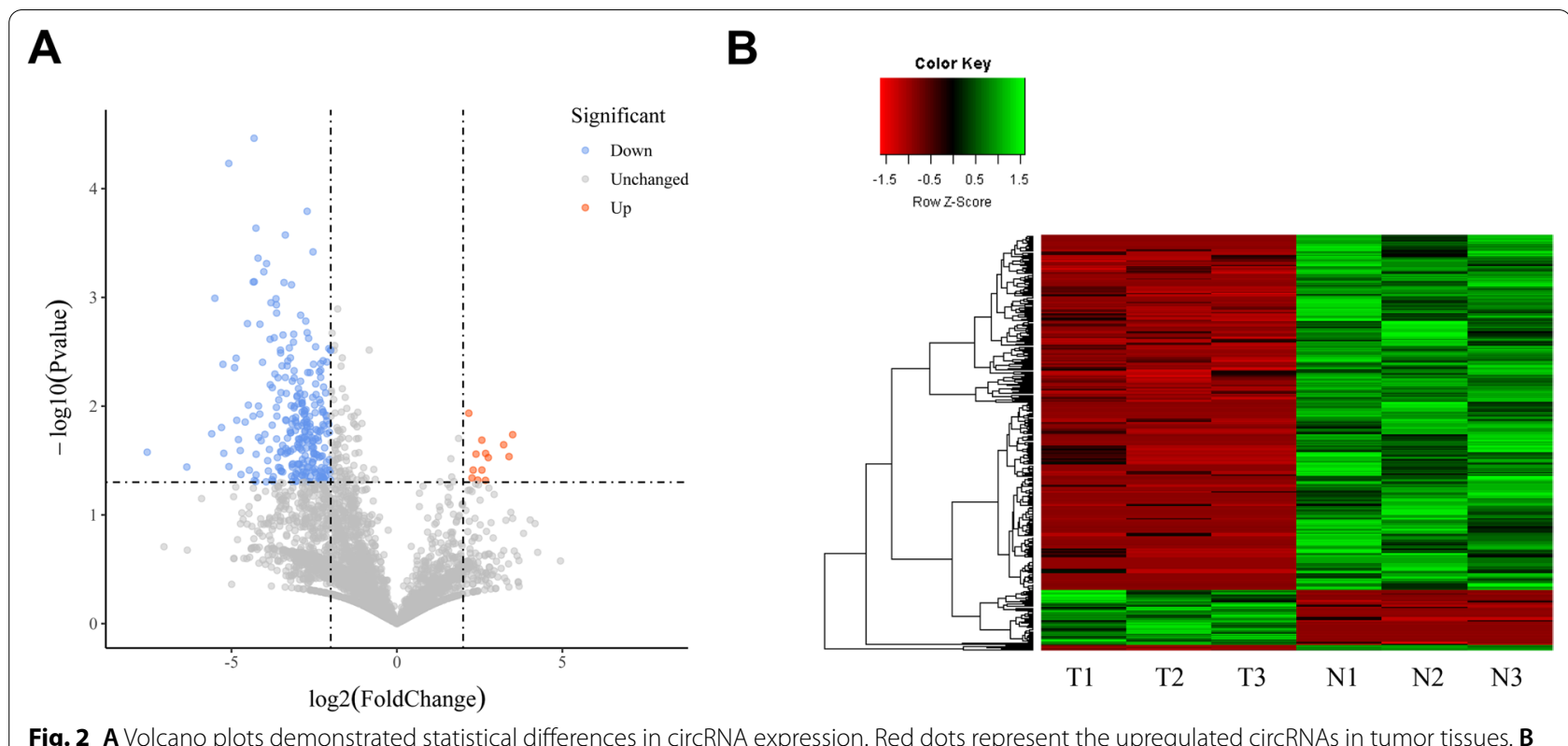

Fig. 2 A Volcano plots demonstrated statistical differences in circRNA expression. Red dots represent the upregulated circRNAs in tumor tissues. B Heatmaps showed the differential expression profile of 353 selected circRNAs in tissues 


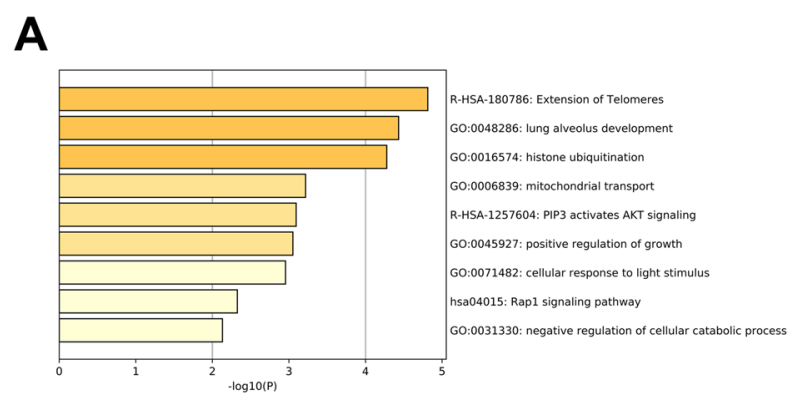

B

C

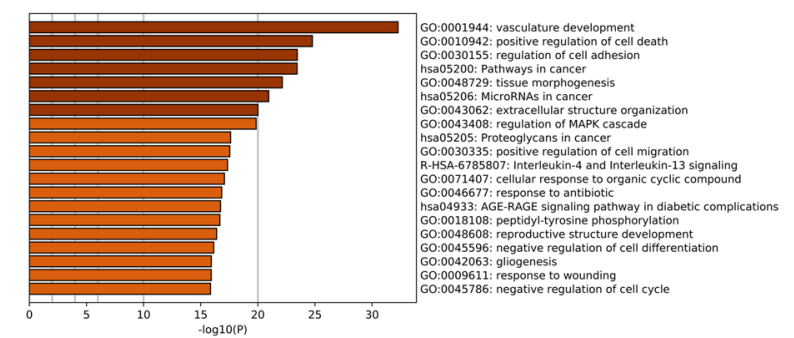

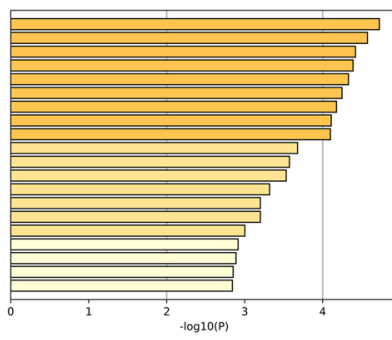

60:0006325: chromatin aromintion 60:0016567. promatin ubiquatinization
G0:0043254: regulation of protein complex assembiy

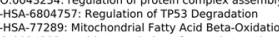
-HSA-77289: Mitochondrial Fatty Acid Beta-0xidation
O O:00334393: positive regulation of smooth muscle cell apoptotic process 60:00136311: dephosphorylation
R-HSA-1640170: Cell Cycle
M101: PID HDAC M1OA1: PID HDAC CLASSI PATHWAY
R-HSA-2990846: SUMOYlation

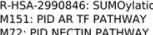
M72: PID NECTIN PATHWAY

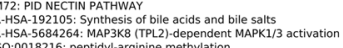
G0:0018216 peptidyl-arginine meethylation
M164: PID ERBBL DOWNSTREAM PATHWAY 10: 14: PID ERBB 1 DOWNSTREAM PATHWAY
60 :007017: microtubule-based process hsa04962: Vasopressin-regulated water reabsorption
M71. PDI IKK PATHWAY
GO:001059: regWtion G0:0010869: regulation of receptor biosynthetic process

D

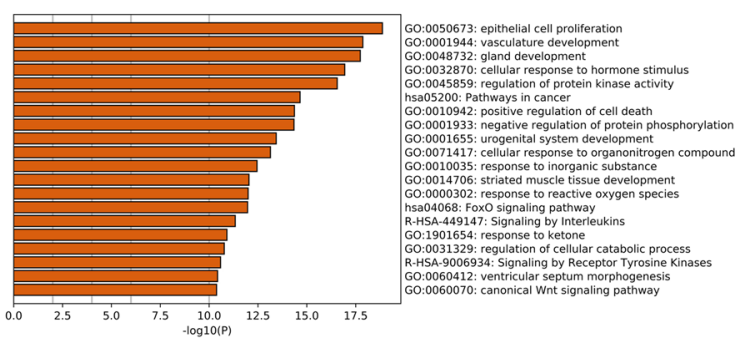

Fig. 3 A Representative enriched terms for circRNAs upregulated in tumor tissues. B Representative enriched terms for circRNAs downregulated in tumor tissues. $\mathbf{C}$ Representative enriched terms for miRNA-target genes upregulated in tumor tissues. D Representative enriched terms for miRNA-target genes downregulated in tumor tissues

expression was associated with poor prognosis. Representative enriched terms of the top 20 clusters in the downregulated group tended to be related to the maintenance of life activities (Fig. 3B). We observed that some enriched terms were related to the normal functions of the human body, such as chromatin organization and protein ubiquitination. The canonical pathway, M164: PID ERBB1 downstream pathway, suggested that para-tumor tissues were likely fighting tumor invasion. A previous study demonstrated that the inhibition of ERBB1 and ERBB2 could rapidly inhibit the motility and intravasation of tumor cells [35]. In such cases, circRNAs of para-tumor tissues have been suggested to play a role in self-regulation for defense. We then constructed a network for enrichment analysis. From this network, we observed that some genes were involved in several terms. Among the top 9 clusters in the upregulated group, KDM1A (also known as LSD1) appeared in 6 terms (Fig. 4A). Among the top 20 representative enriched terms in the downregulated group, EP300 was associated with 11 terms (Fig. 4B). In previous studies, the expression of LSD1 was found to gradually increase from ovarian benign cystadenoma and borderline cystadenoma to cystadenocarcinoma [36]. Likewise, the overexpression of LSD1 mRNA has been observed in human ovarian tumors [37]. Given that the circRNA of KDM1A was also overexpressed in tumor tissues, this gene might be a potential focus for future study.
To explore the potential function of circRNAs in tumor progression, we conducted a conjoint analysis with oncogenes and tumor-suppressor genes. Among the selected circRNAs, we found that 12 parental genes of 14 circRNAs were annotated as oncogenes (Fig. 5A). We also identified 34 tumor-suppressor genes that had circular transcripts (Fig. 5B). We also found that groupspecific circRNAs accounted for the majority of the circRNAs (Fig. 5C, D). This finding further suggested that particular species functioned in tumor progression. For example, the oncogene EPCAM, which had two novel circular isoforms, was only expressed in tumor tissues and is known to contribute to the formation of the protein epithelial cellular adhesion molecule (EpCAM). The human EpCAM protein has been shown to be highly expressed in many samples of human cancer (including ovarian cancer) and is considered a potential immunotherapeutic target for treatment [38].

\section{Co-expression analysis with mRNAs and miRNAs}

Next, we conducted a co-expression analysis with mRNAs and miRNAs to establish the potential relevance. First, we compared the expression patterns of circRNAs and their homologous mRNAs (Fig. 6A), and expression abundance appeared important. We found that the majority of circRNAs and their respective linear transcripts whose expression significantly changed tended to be downregulated in tumor tissues. Only a few of 


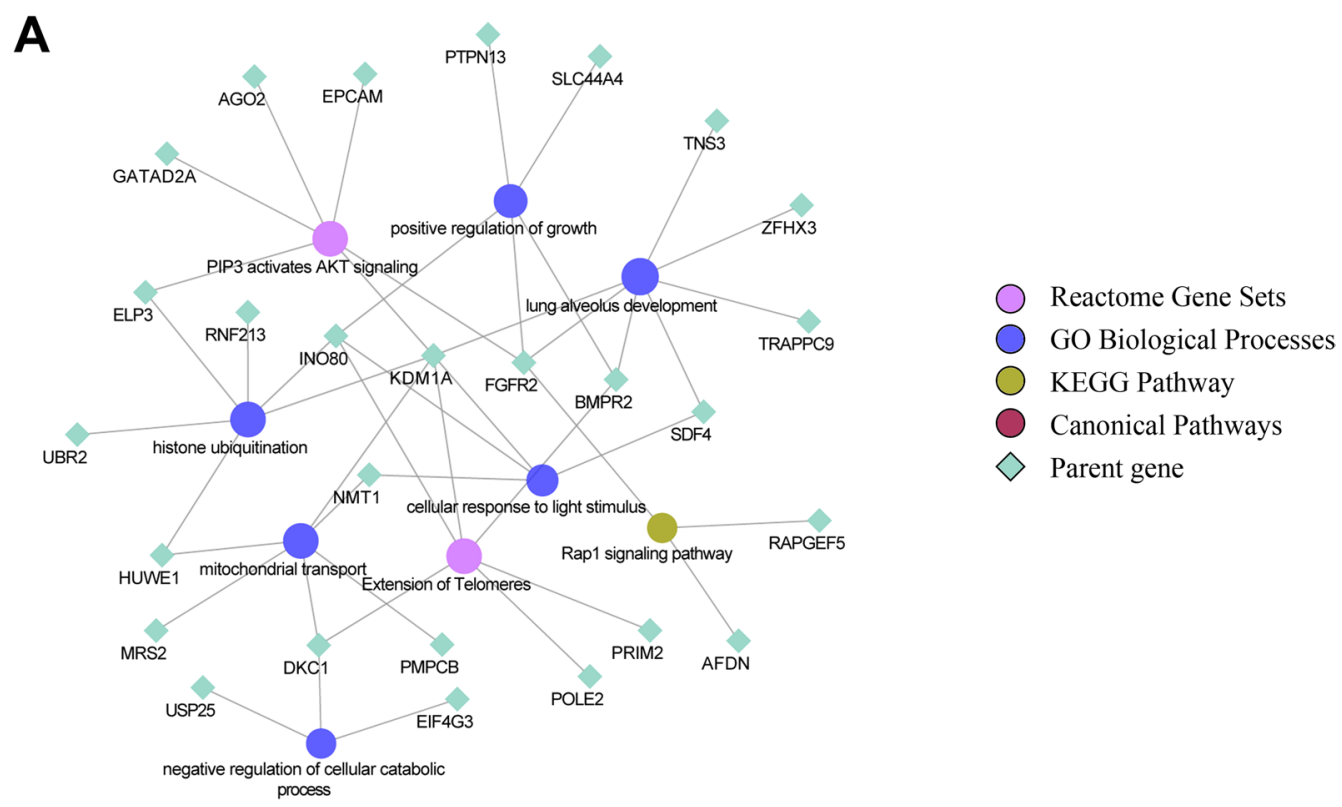

B

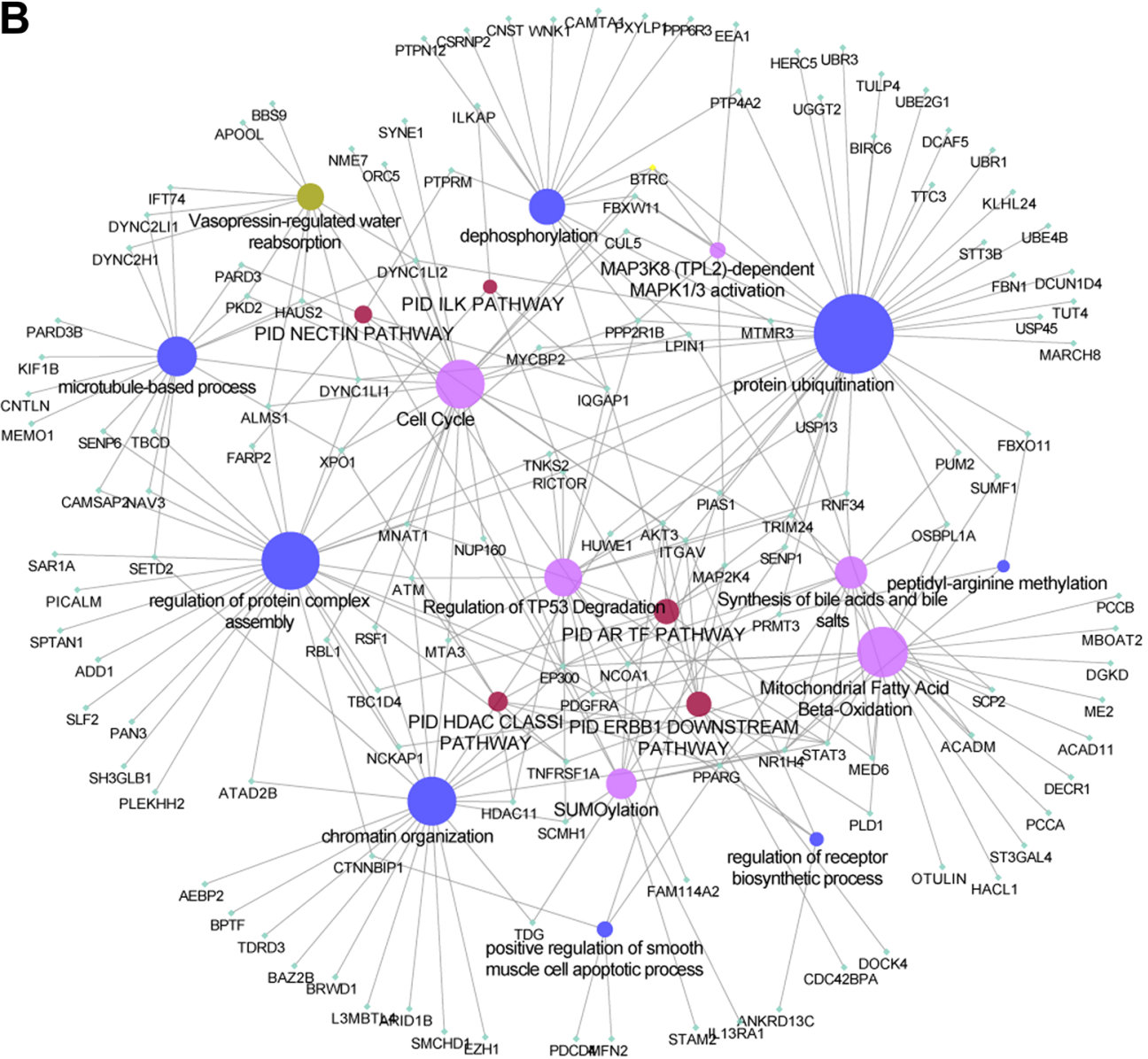

Fig. 4 The network produced by the functional enrichment analysis. The colors and shapes represented different categories, and the size of circular nodes represented the counts of genes for each term. A Pathway network of circRNAs upregulated in tumor tissues. B Pathway network of circRNAs downregulated in tumor tissues 
them showed the opposite pattern of expression. However, some circRNAs exhibited marked changes in their expression level, but the expression level of their mRNAs did not significantly vary. A similar pattern was observed for a portion of the circRNAs that could not be shown in the scatter diagram. We then evaluated the performance of the linear transcripts of oncogenes and tumorsuppressor genes (Fig. 6C, D). And there was overlap among approximately half of the differentially expressed circRNAs derived from oncogenes and tumor-suppressor genes with homologous mRNAs (Fig. 6B).

To further explore the miRNAs, we introduced some miRNAs that were experimentally confirmed to be related to ovarian cancer from miR2Disease. Mapping these miRNAs to the human MTIs from the miRTarBase revealed 1185 miRNA-target genes validated from reporter assays with strong support. We then found that $98.4 \%$ of their linear transcripts appeared in the tissues, and $434(36.62 \%)$ were significantly expressed by characterization of the fold-change (Fig. 7A). Functional analysis classified by variation trend was then conducted, and darker colors of the representative enriched terms corresponded to lower $p$-values (Fig. 3C, D). Pathways linked to cancer were obvious in both groups. For the downregulated groups, biological processes, such as epithelial cell proliferation, vasculature development, and gland development, indicated that they functioned through self-regulation in para-tumor tissues. Enriched terms including signaling by interleukins and signaling by receptor tyrosine kinases reflected their potential as therapeutic targets for ovarian cancer. For example, inhibition of interleukin 6 (IL-6) was found to facilitate therapeutic activity when the anti-IL-6 antibody siltuximab was taken in clinical trials of patients with platinum-resistant ovarian cancer [39]. The receptor-specific tyrosine kinase inhibitor ZD 1839 ('Iressa') also contributed to the growth inhibition in ovarian cell lines [40]. For the upregulated group, specific biological processes such as response to wounding, positive regulation of cell migration, negative regulation of cell cycle, and positive regulation of cell death demonstrated the pressure of tumor progression in tissues. Furthermore, 33 miRNA-target genes had relevant circRNAs that were found to be differentially expressed in tissues. A total of $45.45 \%$ of these genes were differentially expressed by characterization of the fold-change, and most of them were downregulated in tumor tissues (Fig. 7C). The expression of most of these genes was consistent with the patterns of circRNA expression. Next, the interaction between miRNAs and differentially expressed circRNAs was predicted by miRanda. As mentioned previously, 353 differentially expressed circRNAs (46 upregulated and 307 downregulated) were used for prediction. A total of 261 of them (73.94\%) were predicted to target miRNAs related to ovarian cancer, including 32 upregulated and 229 downregulated circRNAs (Fig. 7B). We then calculated the numbers of miRNAs that each circRNAs targeted. Using 16 as the maximum number, circRNAs targeting over 5 miRNAs were used to construct a circRNA-miRNA co-expression network. We used Cytoscape (version 3.6.1) to visualize the complex network (Fig. 8) [41]. In this network, identified circRNAs that were not recorded in circBase were annotated with symbols of their parent genes. A total of 30 circRNAs and 82 disease-related miRNAs (appeared as nodes) were used to establish 235 edges to organize the network, which clearly illustrated their regulatory relationships and the associated pathways.

\section{Discussion}

Ovarian serous cystadenocarcinoma is a type of ovarian epithelial malignancy [2]. Because of the difficulty associated with early diagnosis and treatment, exploring the features at the molecular level is important. Recently, researchers have noticed that the dysregulation of circRNAs might underlie some diseases [11-13]. However, the association between circRNAs and ovarian serous cystadenocarcinoma remains unclear. Here, we explored the expression profile of circRNAs in ovarian serous cystadenocarcinoma.

In this study, we successfully identified many circRNAs in tumor and normal tissues and then conducted an analysis to characterize the differential distribution of these circRNAs. Although the two groups were roughly equal in the number of circRNAs, the distribution of back-spliced reads and quantification demonstrated the marked change in the expression level between the two groups. We detected 353 differentially expressed circRNAs and observed the patterns of expression between tissues. Overall, more circRNAs were weakly expressed in tumor tissues of ovarian serous cystadenocarcinoma. We found that some circRNAs were only significantly expressed in all three specimens of either tumor or normal tissues. These group-specific circRNAs accounted for nearly $59 \%$ of the differentially expressed circRNAs. In addition, these circRNAs potentially functioned in tumor progression based on their proportion determined

(See figure on next page.)

Fig. 5 A Occurrence of oncogenes in differentially expressed circRNAs. B Occurrence of tumor-suppressor genes in differentially expressed circRNAs. C Distribution of oncogenes in differentially expressed circRNAs in tissues. "Group-specific" meant that some differentially expressed circRNAs were only significantly expressed in all three specimens of one group and were not expressed in the other group. D Distribution of tumor-suppressor genes in differentially expressed circRNAs in different tissues 
A

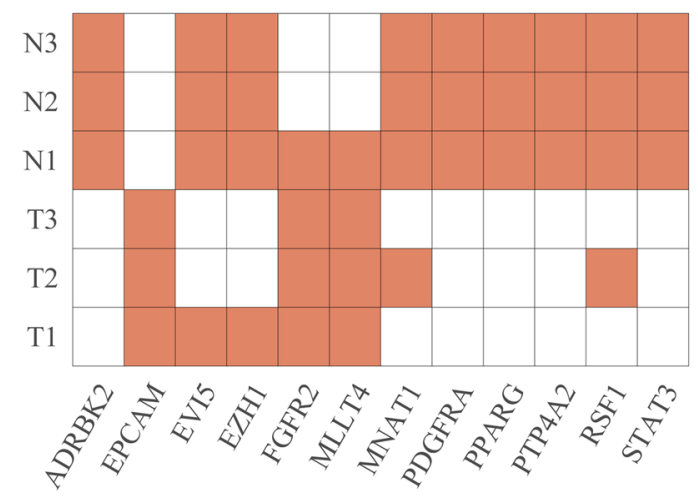

B

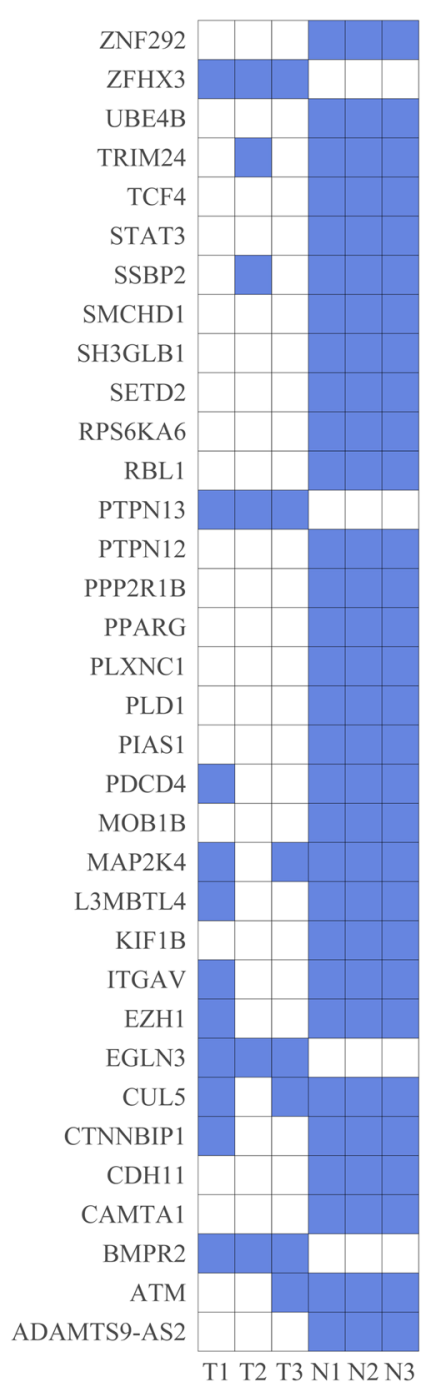

C

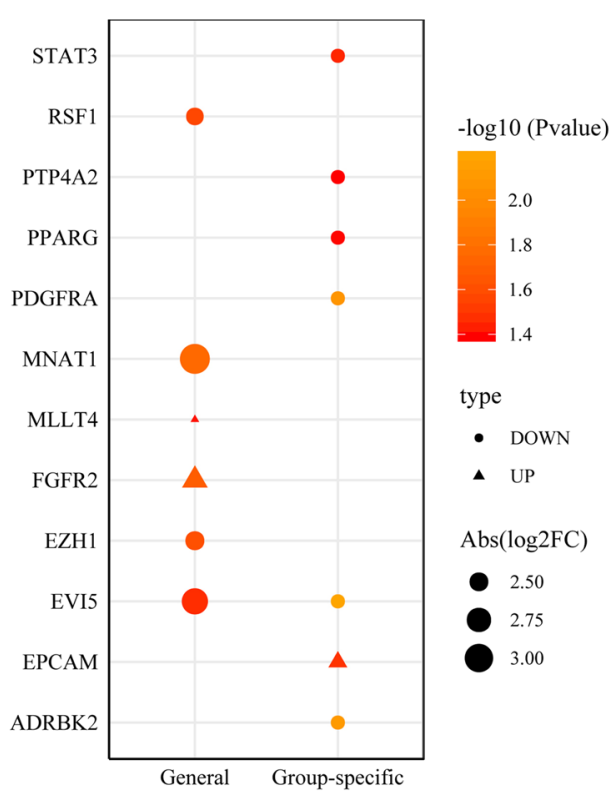

D

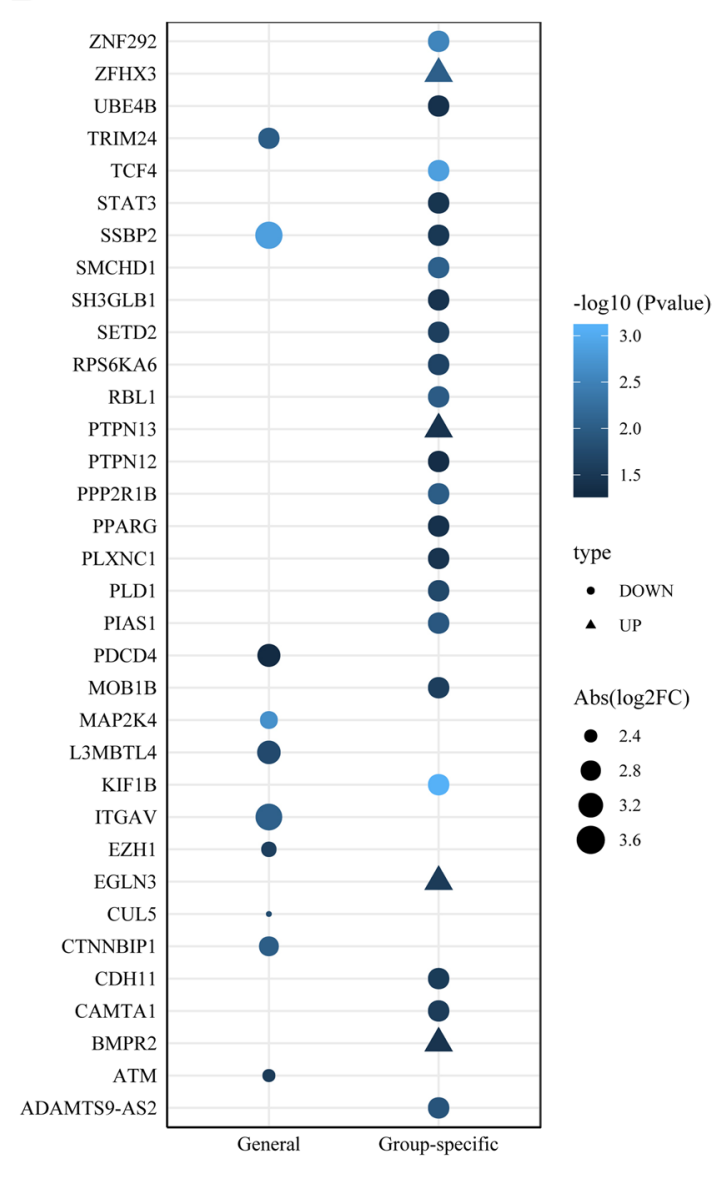

Fig. 5 (See legend on previous page.) 
A

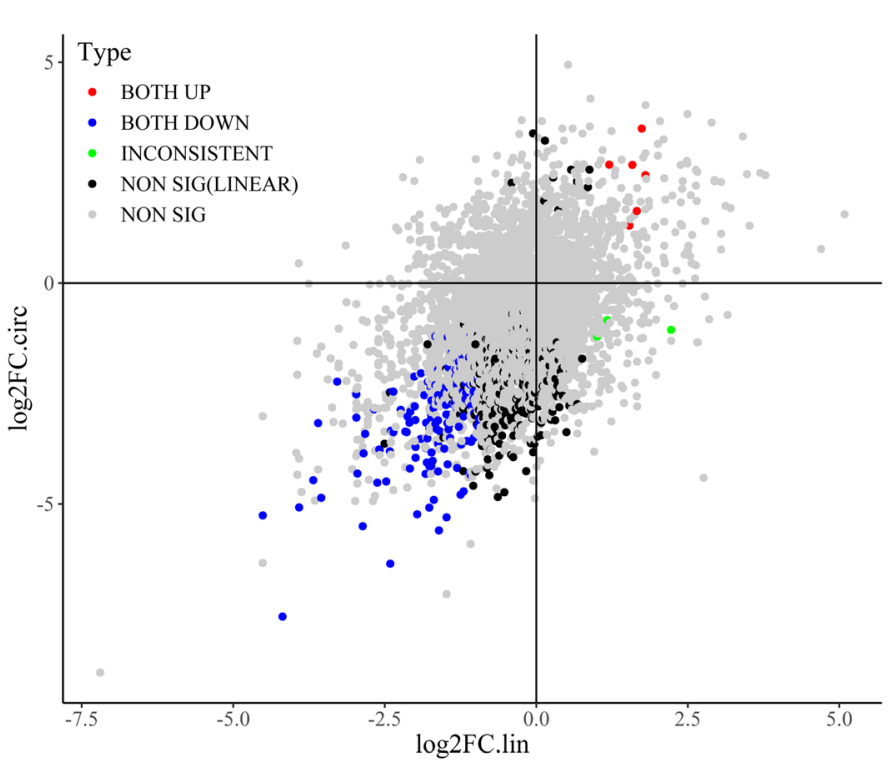

B

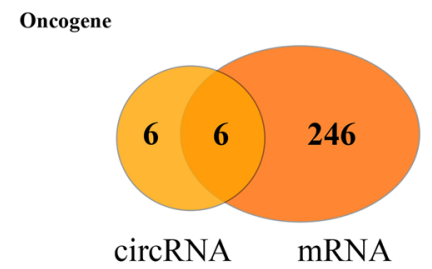

Tumor-suppressor gene

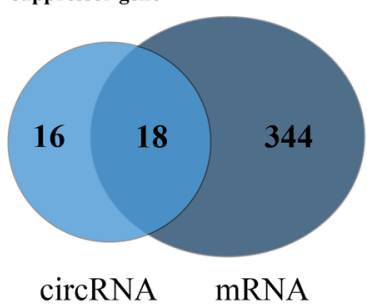

C

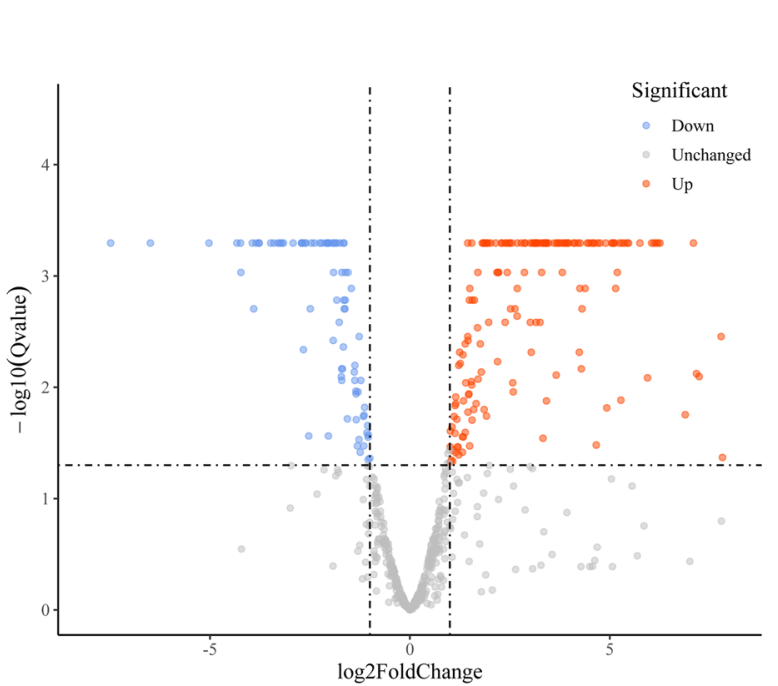

D

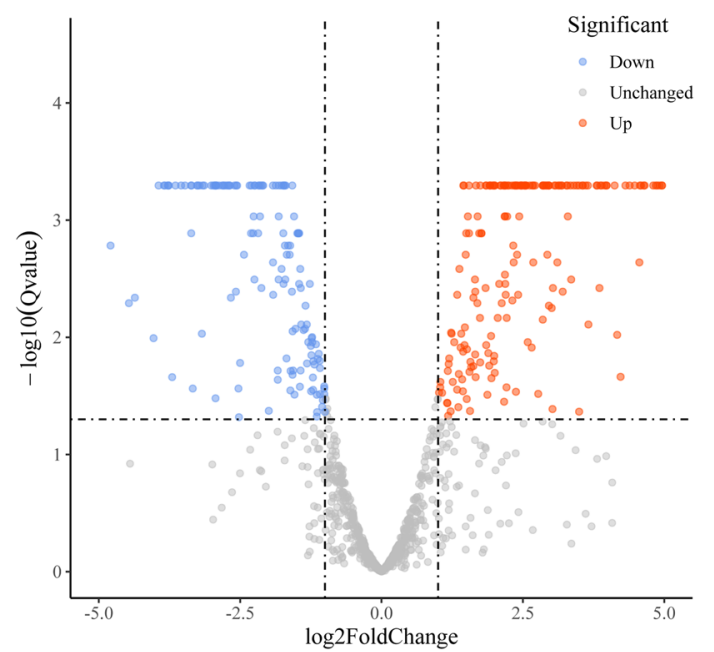

Fig. 6 A The correlation between the expression of circRNAs and their homologous mRNAs measured by log2Foldchange. The red and blue points indicated that circRNAs and their linear transcripts were either significantly upregulated or downregulated in tumor tissues, respectively. Green points indicated that circRNAs and their linear transcripts had different patterns of change. However, some circRNAs (black points) were expressed differentially when their respective mRNAs did not show parallel changes. B Overlap in the occurrence of oncogenes and tumor-suppressor genes between differentially expressed circRNAs and mRNAs. C Distribution of oncogenes in differentially expressed mRNAs in tissues. D Distribution of tumor-suppressor genes in differentially expressed mRNAs in tissues

by the analysis with oncogenes and tumor-suppressor genes. Under the stimulation of cancerous process in tissues, the expression of circRNAs involved in tumor progression might change dramatically.

In the enrichment analysis, circRNAs active in the tumor tissues were associated with tumor development, whereas those in the para-tumor tissues were not. We found that 2 of the genes involved in the top 9 clusters in the upregulated group, EPCAM and FGFR2, were oncogenes. Both of these genes were associated with the term R-HSA-1257604: PIP3 activates AKT signaling. This pathway was once thought to be associated with ovarian serous cystadenocarcinoma. Meanwhile, the gene KDM1A, whose products are active in ovarian cancer 


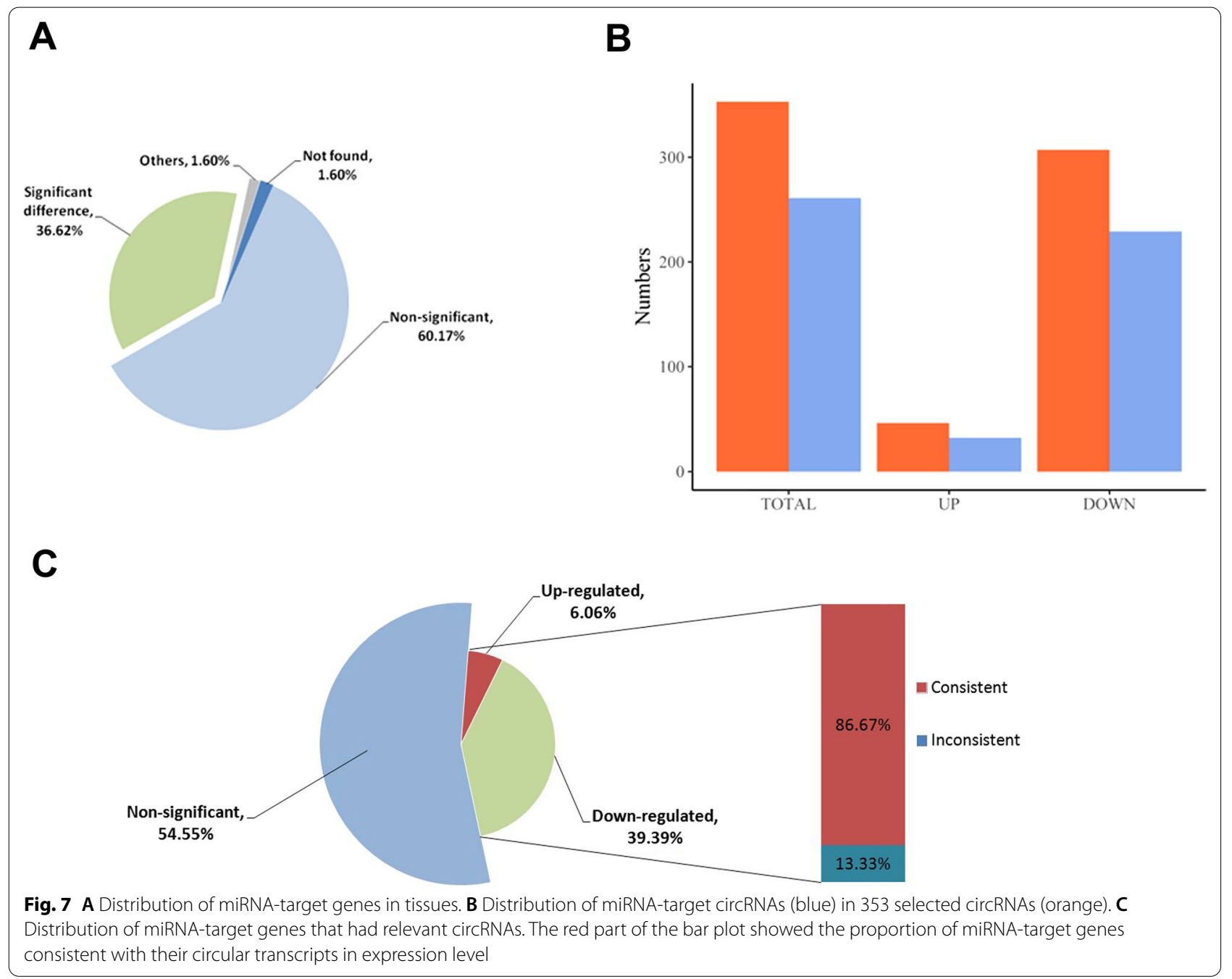

cells, also appeared in this pathway. Therefore, the pathway R-HSA-1257604: PIP3 activates AKT signaling might be an important pathway in the development of ovarian serous cystadenocarcinoma.

To further explore the function of circRNAs, we attempted to conduct a co-expression analysis with mRNAs and miRNAs. First, we explored the relationship between circRNAs and their parent genes. Given that the production of circRNAs is involved in spliceosomes, circRNAs were thought to compete with their homologous mRNAs for splice sites [15]. The promotion of flanking intronic sequences for circularization might lead to a lower linear splicing efficiency of flanking exons. However, the correlation in this study was the opposite. In addition, this result was obtained based on a screening analysis for the special target genes and their circular isoforms. This consistency was also observed in the analysis with oncogenes and tumor-suppressor genes. For example, circRNAs of the oncogene EPCAM were only expressed in tumor tissues, whereas the expression of relevant mRNAs exhibited a 25 -fold increase. In terms of tumor-suppressor genes, MAP2K4 inactivation was confirmed in ovarian cancer cell lines [42]. We also observed clear upregulation of the circRNAs and mRNAs of MAP2K4 in para-tumor tissues. Considering the marked increase in expression level, we hypothesized that competition between transcripts became weaker because of the tumorigenesis in ovarian serous cystadenocarcinoma. Based on the co-expression analysis, our prediction suggested a strong interaction between circRNAs and miRNAs related to ovarian cancer. We noted that miR-608 was targeted by 8 circRNAs upregulated in tumor tissues. miR-608 was thought to inhibit tumor proliferation and migration by targeting special proteins in lung adenocarcinoma and hepatocellular carcinoma $[43,44]$. These upregulated circRNAs could thus indirectly contribute to the invasion of ovarian serous cystadenocarcinoma. Additionally, we constructed a complex 


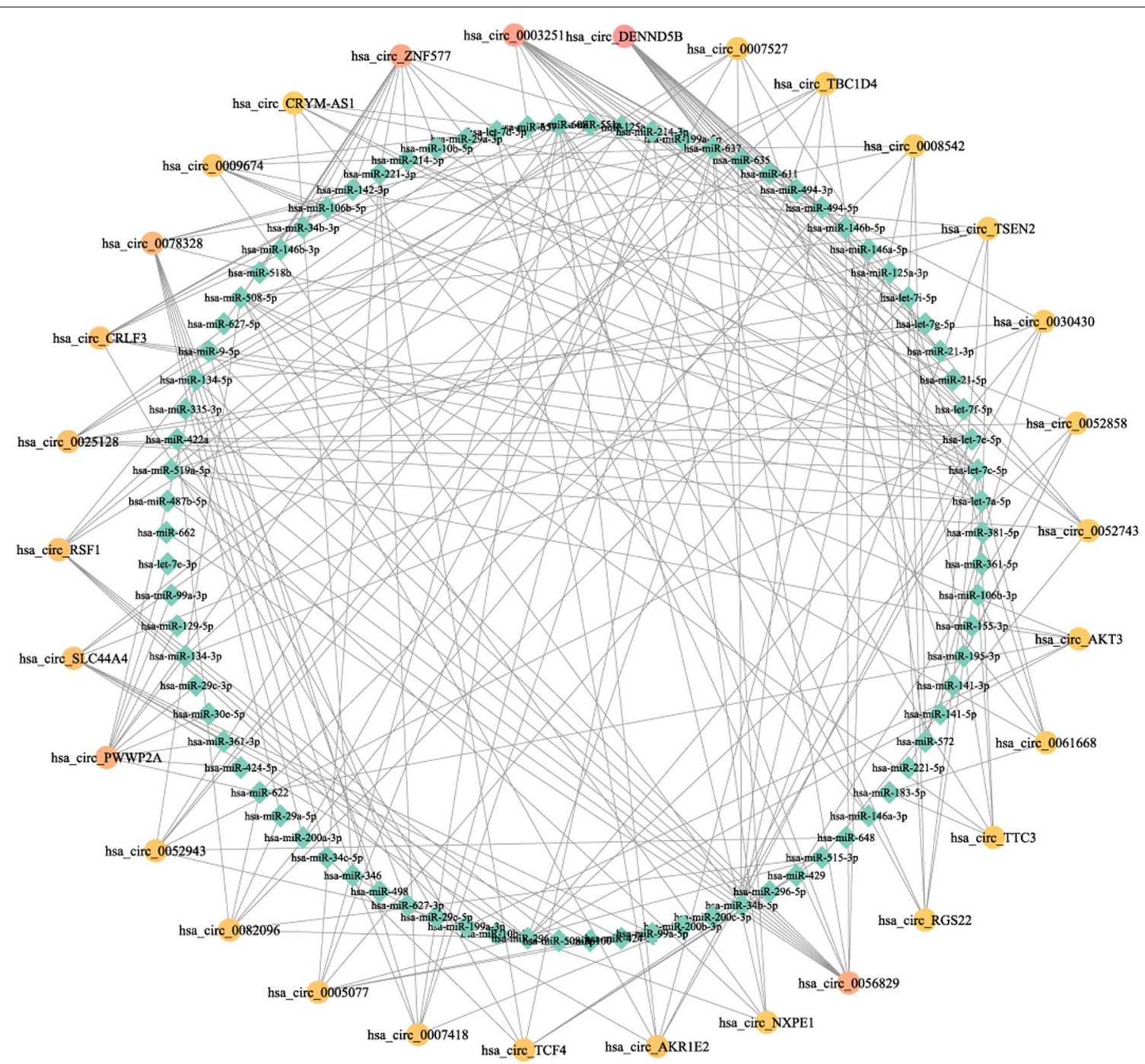

Fig. 8 Co-expression network between miRNAs and circRNAs. The rhombic nodes represented miRNAs, and the circular nodes represented circRNAs. The change in circular nodes from yellow to red revealed an increase in the number of targeted miRNAs

network to select potential biomarkers in circRNAs. circRNAs can remain stable in cells because their circular structure is more resistant to exonucleolytic activities [45]. This property indicates that circRNAs could be used in clinical diagnosis. Based on the network, half of these circRNAs (15 of 30), which had strong connections with the selected miRNAs, were novel, including hsa_circ DENND5B. Hsa_circ_DENND5B was found to target 16 miRNAs relevant to ovarian cancer, which was the largest number of miRNAs for a single circRNA. Meanwhile, hsa_circ_0003251 ranked second in the relevant number (14) and was considered a potential miRNA sponge in a previous study examining diabetes and depression [46]. However, hsa_circ_SLC44A4 and hsa_circ_AKR1E2, separately related to 8 and 7 miRNAs, respectively, were noteworthy given their level of enrichment in tumor tissues. miRNAs that hsa_circ_AKR1E2 targeted included miR-608 and 3 miRNAs of the let-7 family. A previous study has suggested that members of the let-7 family can act as tumor suppressors in multiple carcinomas [47]. Therefore, we speculate that these 4 aforementioned circRNAs could be used as potential biomarkers in response to ovarian serous cystadenocarcinoma.

Here, we conducted a multi-angle analysis for circRNAs in ovarian serous cystadenocarcinoma. Several limitations of our study require consideration. The number of specimens was small and did not contain the normal tissues from volunteers free of ovarian serous cystadenocarcinoma. Nevertheless, our study established the expression profile of circRNAs in ovarian 
serous cystadenocarcinoma and provides a foundation for future studies to validate these findings.

\section{Conclusions}

In this study, we characterized the expression profile of circRNAs in ovarian serous cystadenocarcinoma and conducted a co-expression analysis with mRNAs and miRNAs. We identified 15,092 unique circRNAs and clarified their patterns of differential expression in tissues. Enrichment analysis and prediction of the interactions with 353 differentially expressed circRNAs indicated that the function of circRNAs was to facilitate tumor progression, suggesting the presence of a potential pathway in ovarian serous cystadenocarcinoma. Moreover, consistent changes between circRNAs and the homologous mRNAs might be associated with latent regulation in ovarian serous cystadenocarcinoma. After network analysis, several circRNAs were determined to represent possible biomarkers. Generally, our study established the role of circRNAs in the tumorigenesis of ovarian serous cystadenocarcinoma and provides a basis for future work.

\section{Abbreviations}

circRNAs: Circular RNAs; miRNA: MicroRNA; GO: Gene ontology; pre-mRNA: Precursor mRNA; polyA: Polyadenylated; MTIs: MiRNA-target interactions; RPM: Reads per million mapped reads; PAKT: Phosphorylated AKT.

\section{Supplementary Information}

The online version contains supplementary material available at https://doi. org/10.1186/s12920-021-01132-5.

Additional file 1: Fig. S1. The sequence depths of six specimens.

\section{Acknowledgements}

Not applicable.

\section{About this supplement}

This article has been published as partof BMC Medical Genomics Volume 14 Supplement 2 2021: Data-drivenanalytics in biomedical genomics (part 2). The full contents of thesupplement are available athttps://bmcmedgenomics. biomedcentral.com/articles/supplements/volume-14-supplement-2.

\section{Authors' contributions}

$\mathrm{XS}, \mathrm{PH}$, and SF conceived the study and revised the manuscript. MZ implemented the study, analyzed the data, and wrote the manuscript; JZ and JW assisted with the data analysis. All authors read and approved the final version of the manuscript.

\section{Funding}

This work was supported by the National Natural Science Foundation of China (No. 61973155), the National Natural Science Foundation of China (No. 62003165), and the China Postdoctoral Science Foundation (No. 2019M661817). Publication costs were funded by the National Natural Science Foundation of China (No. 61973155). The funding bodies played no role in study design, data collection and analysis, or preparation of the manuscript.

\section{Availability of data and materials}

The sequencing data have been deposited in the GSA-Human datasets of the National Genomics Data Center in China (https://bigd.big.ac.cn/) under the accession number PRJCA002938.

\section{Declarations}

\section{Ethics approval and consent to participate}

All patients were informed of the purpose of the study and signed informed consents, and the collection of specimens was approved by the ethics committee of Jiangsu Province Hospital (2015-SRFA-121).

\section{Consent for publication}

Specimens from human ovarian tissues were obtained with written informed consent. All data were anonymized, and no information was traceable to any individual donor.

\section{Competing interests}

The authors declare no competing interests.

\section{Author details}

${ }^{1}$ Department of Biomedical Engineering, Nanjing University of Aeronautics and Astronautics, Nanjing 211106, China. ${ }^{2}$ School of Biomedical Engineering and Informatics, Nanjing Medical University, Nanjing 211166, China. ${ }^{3}$ Department of Gynecology and Obstetrics, The First Affiliated Hospital With Nanjing Medical University, Nanjing 210029, China.

Received: 3 November 2021 Accepted: 16 November 2021

Published: 2 December 2021

\section{References}

1. Siegel RL, Miller KD, Jemal A. Cancer statistics, 2018: cancer statistics, 2018. CA Cancer J Clin. 2018;68(1):7-30.

2. Kawamoto S, Urban BA, Fishman EK. CT of epithelial ovarian tumors. Radio Graph. 1999;19(1):85-102.

3. Alsop K, Fereday S, Meldrum C, deFazio A, Emmanuel C, George J, et al. BRCA mutation frequency and patterns of treatment response in BRCA mutation-positive women with ovarian cancer: a report from the australian ovarian cancer study group. J Clin Oncol. 2012;30(21):2654-63.

4. Bali A, O'Brien PM, Edwards LS, Sutherland RL, Hacker NF, Henshall SM. Cyclin D1, p53, and p21Waf1/Cip1 expression is predictive of poor clinical outcome in serous epithelial ovarian cancer. Clin Cancer Res. 2004;10(15):5168-77.

5. Hefler LA, Zeillinger R, Grimm C, Sood AK, Cheng WF, Gadducci A, et al. Preoperative serum vascular endothelial growth factor as a prognostic parameter in ovarian cancer. Gynecol Oncol. 2006;103(2):512-7.

6. Sanger HL, Klotz G, Riesner D, Gross HJ, Kleinschmidt AK. Viroids are single-stranded covalently closed circular RNA molecules existing as highly base-paired rod-like structures. Proc Natl Acad Sci U S A. 1976;73(11):3852-6.

7. Hsu M-T, Coca-Prados M. Electron microscopic evidence for the circular form of RNA in the cytoplasm of eukaryotic cells. Nature. 1979;280(5720):339

8. Nigro JM, Cho KR, Fearon ER, Kern SE, Ruppert JM, Oliner JD, et al. Scrambled exons. Cell. 1991;64(3):607-13.

9. Enuka Y, Lauriola M, Feldman ME, Sas-Chen A, Ulitsky I, Yarden Y. Circular RNAs are long-lived and display only minimal early alterations in response to a growth factor. Nucleic Acids Res. 2016;44(3):1370-83.

10. Zhang Y, Xue W, Li X, Zhang J, Chen S, Zhang J-L, et al. The biogenesis of nascent circular RNAs. Cell Rep. 2016;15(3):611-24.

11. Bachmayr-Heyda A, Reiner AT, Auer K, Sukhbaatar N, Aust S, BachleitnerHofmann T, et al. Correlation of circular RNA abundance with proliferation-exemplified with colorectal and ovarian cancer, idiopathic lung fibrosis, and normal human tissues. Sci Rep. 2015;5:8057.

12. Xu Y, Yao Y, Zhong X, Leng K, Qin W, Qu L, et al. Downregulated circular RNA hsa_circ_0001649 regulates proliferation, migration and 
invasion in cholangiocarcinoma cells. Biochem Biophys Res Commun. 2018;496(2):455-61.

13. Yu J, Xu Q-G, Wang Z-G, Yang Y, Zhang L, Ma J-Z, et al. Circular RNA CSMARCA5 inhibits growth and metastasis in hepatocellular carcinoma. J Hepatol. 2018;68(6):1214-27.

14. Ning L, Long B, Zhang W, Yu M, Wang S, Cao D, et al. Circular RNA profiling reveals circEXOC6B and circN4BP2L2 as novel prognostic biomarkers in epithelial ovarian cancer. Int J Oncol. 2018;53(6):2637-46.

15. Ashwal-Fluss R, Meyer M, Pamudurti NR, Ivanov A, Bartok O, Hanan M, et al. circRNA biogenesis competes with pre-mRNA splicing. Mol Cell. 2014;56(1):55-66.

16. Hansen TB, Jensen TI, Clausen BH, Bramsen JB, Finsen B, Damgaard CK et al. Natural RNA circles function as efficient microRNA sponges. Nature. 2013;495(7441):384-8.

17. Memczak S, Jens M, Elefsinioti A, Torti F, Krueger J, Rybak A, et al. Circular RNAs are a large class of animal RNAs with regulatory potency. Nature. 2013;495(7441):333-8.

18. Hansen TB, Kjems J, Damgaard CK. Circular RNA and miR-7 in cancer. Cancer Res. 2013;73(18):5609-12.

19. Yeh Y-M, Chuang C-M, Chao K-C, Wang L-H. MicroRNA-138 suppresses ovarian cancer cell invasion and metastasis by targeting SOX 4 and HIF-1a. Int J Cancer. 2013;133(4):867-78.

20. Li H. Aligning sequence reads, clone sequences and assembly contigs with BWA-MEM. arXiv: 13033997 [q-bio] [Internet]. 2013 May 26; Available from: http://arxiv.org/abs/1303.3997.

21. Kent WJ, Sugnet CW, Furey TS, Roskin KM, Pringle TH, Zahler AM, et al. The human genome browser at UCSC. Genome Res. 2002;12(6):996-1006.

22. Gao Y, Wang J, Zhao F. CIRI: an efficient and unbiased algorithm for de novo circular RNA identification. Genome Biol. 2015;16(1):4.

23. Gao Y, Zhang J, Zhao F. Circular RNA identification based on multiple seed matching. Brief Bioinform. 2018:19(5):803-10.

24. Kim D, Pertea G, Trapnell C, Pimentel H, Kelley R, Salzberg SL. TopHat2: accurate alignment of transcriptomes in the presence of insertions, deletions and gene fusions. Genome Biol. 2013;14(4):R36.

25. Trapnell C, Roberts A, Goff L, Pertea G, Kim D, Kelley DR, et al. Differential gene and transcript expression analysis of RNA-seq experiments with TopHat and Cufflinks. Nat Protoc. 2012;7(3):562-78.

26. Glažar P, Papavasileiou P, Rajewsky N. circBase: a database for circular RNAs. RNA. 2014;20(11):1666-70.

27. Jiang $Q$, Wang $Y$, Hao Y, Juan $L$, Teng $M$, Zhang $X$, et al. miR2Disease: a manually curated database for microRNA deregulation in human disease. Nucleic Acids Res. 2009:37(Database issue):D98-104.

28. Chou C-H, Shrestha S, Yang C-D, Chang N-W, Lin Y-L, Liao K-W, et al. miRTarBase update 2018: a resource for experimentally validated microRNAtarget interactions. Nucleic Acids Res. 2018;46(Database issue):D296-302.

29. Liu Y, Sun J, Zhao M. ONGene: a literature-based database for human oncogenes. J Genet Genom. 2017:44(2):119-21.

30. Zhao M, Kim P, Mitra R, Zhao J, Zhao Z. TSGene 2.0: an updated literaturebased knowledgebase for tumor suppressor genes. Nucleic Acids Res. 2016;44(D1):D1023-31.

31. Zhou Y, Zhou B, Pache L, Chang M, Khodabakhshi AH, Tanaseichuk O, et al. Metascape provides a biologist-oriented resource for the analysis of systems-level datasets. Nat Commun. 2019;10(1):1523.
32. Betel D, Koppal A, Agius P, Sander C, Leslie C. Comprehensive modeling of microRNA targets predicts functional non-conserved and non-canonical sites. Genome Biol. 2010;11(8):R90.

33. Dobbin ZC, Landen CN. The importance of the PI3K/AKT/MTOR pathway in the progression of ovarian cancer. Int J Mol Sci. 2013;14(4):8213-27.

34. Huang Y, Hua K, Zhou X, Jin H, Chen X, Lu X, et al. Activation of the PI3K AKT pathway mediates FSH-stimulated VEGF expression in ovarian serous cystadenocarcinoma. Cell Res. 2008;18(7):780-91.

35. Kedrin D, Wyckoff J, Boimel PJ, Coniglio SJ, Hynes NE, Arteaga CL, et al. ERBB1 and ERBB2 have distinct functions in tumor cell invasion and intravasation. Clin Cancer Res. 2009;15(11):3733-9.

36. Chen C, Ge J, Lu Q, Ping G, Yang C, Fang X. Expression of lysine-specific demethylase 1 in human epithelial ovarian cancer. J Ovarian Res. 2015;8(1):28

37. Konovalov S, Garcia-Bassets I. Analysis of the levels of lysine-specific demethylase 1 (LSD1) mRNA in human ovarian tumors and the effects of chemical LSD1 inhibitors in ovarian cancer cell lines. J Ovarian Res. 2013;6(1):75.

38. Baeuerle PA, Gires O. EpCAM (CD326) finding its role in cancer. Br J Cancer. 2007:96(3):417-23.

39. Coward J, Kulbe H, Chakravarty P, Leader D, Vassileva V, Leinster DA, et al. Interleukin-6 as a therapeutic target in human ovarian cancer. Clin Cancer Res. 2011;17(18):6083-96.

40. Sewell JM, Macleod KG, Ritchie A, Smyth JF, Langdon SP. Targeting the EGF receptor in ovarian cancer with the tyrosine kinase inhibitor ZD 1839 ('Iressa'). Br J Cancer. 2002;86(3):456-62.

41. Shannon P, Markiel A, Ozier O, Baliga NS, Wang JT, Ramage D, et al. Cytoscape: a software environment for integrated models of biomolecular interaction networks. Genome Res. 2003;13(11):2498-504.

42. Davis SJ, Choong DY, Ramakrishna M, Ryland GL, Campbell IG, Gorringe $\mathrm{KL}$. Analysis of the Mitogen-activated protein kinase kinase 4 (MAP2K4) tumor suppressor gene in ovarian cancer. BMC Cancer. 2011;11(1):173.

43. He L, Meng D, Zhang S-H, Zhang Y, Deng Z, Kong L-B. microRNA-608 inhibits human hepatocellular carcinoma cell proliferation via targeting the BET family protein BRD4. Biochem Biophys Res Commun. 2018;501(4):1060-7.

44. Yu H-X, Wang X-M, Han X-D, Cao B-F. MiR-608 exerts tumor suppressive function in lung adenocarcinoma by directly targeting MIF. Eur Rev Med Pharmacol Sci. 2018;22(15):4908-16.

45. Jeck WR, Sorrentino JA, Wang K, Slevin MK, Burd CE, Liu J, et al. Circular RNAs are abundant, conserved, and associated with ALU repeats. RNA. 2013;19(2):141.

46. Jiang G, Ma Y, An T, Pan Y, Mo F, Zhao D, et al. Relationships of circular RNA with diabetes and depression. Sci Rep. 2017;7(1):7285.

47. Roush S, Slack FJ. The let-7 family of microRNAs. Trends Cell Biol. 2008;18(10):505-16.

\section{Publisher's Note}

Springer Nature remains neutral with regard to jurisdictional claims in published maps and institutional affiliations.

Ready to submit your research? Choose BMC and benefit from

- fast, convenient online submission

- thorough peer review by experienced researchers in your field

- rapid publication on acceptance

- support for research data, including large and complex data types

- gold Open Access which fosters wider collaboration and increased citations

- maximum visibility for your research: over $100 \mathrm{M}$ website views per year

At $\mathrm{BMC}$, research is always in progress.

Learn more biomedcentral.com/submissions 